\title{
Picomole per Ten Billion Cells
}

National Cancer Institute

\section{Source}

National Cancer Institute. Picomole per Ten Billion Cells. NCI Thesaurus. Code C122223.

A unit of concentration (molarity unit) equal to one picomole of substance per ten to the tenth power cells. 